\title{
Buckling analysis of graphene nanosheets by the finite element method
}

\author{
Jozef Bocko ${ }^{1}$, Pavol Lengvarský ${ }^{1, *}$ \\ ${ }^{1}$ Department of Applied Mechanics and Mechanical Engineering, Faculty of Mechanical Engineering, \\ Technical University of Košice, Letná 9, 04200 Košice, Slovakia
}

\begin{abstract}
The paper is devoted to the problems related to buckling analysis of graphene sheets without and with vacancies in the structure under different boundary conditions. The analysis was performed by the classical numerical treatment - the finite element method (FEM). The graphene sheets were modelled by beam elements. Interatomic relations between carbon atoms in the structure were represented by the beams connecting individual atoms. The behaviour of the beam as structural element was based on the properties that were established from relations of molecular mechanics. The vacancies in single layer graphene sheets (SLGSs) were created by elimination of randomly chosen atoms and corresponding beam elements connected to the atoms in question. The computations were accomplished for different percentage of atom vacancies and the results represent an obvious fact that the critical buckling force decreases for increased percentage of vacancies in the structure. The numerical results are represented in form of graphs.
\end{abstract}

Keywords: buckling, graphene sheet, vacancy, beam, finite element method

\section{Introduction}

A carbon is a chemical element with symbol $C$, it is a structural element of many structures. The atoms of carbon can bond together in different ways as graphite, diamond, graphene, etc. Graphene is a thin two-dimensional layer of carbon atoms. It has remarkable mechanical properties and many potential applications of these properties in the future. Among the remarkable mechanical properties belong high Young's modulus, shear modulus, strength, etc. These properties are high only for graphene sheets without vacancies or various crystallographic defects and therefore many researchers study effect of these defects on the mechanical properties.

Researchers started study the elastic properties of graphene sheets as Sakhaee-Pour [1]. He investigated the elastic characteristics (Young's modulus, shear modulus, Poisson's ratio) of defect-free single-layered graphene sheet. The graphene sheets were modelled as a beam finite element model. Georgantzinos et al. [2] computed the elastic material properties of graphene using spring finite element model and they concluded that the numerical results of the Young's modulus, shear modulus and Poisson's ratio for the graphene structures depend on their chilarity, width, length and number of layers. Ansari et

* Corresponding author: pavol.lengvarsky@tuke.sk

Reviewers: Milan Nad', Milan Sága 
al. [3] investigated two types of structural defects (Stone-Wales and single vacancy) on the mechanical properties of single-layered graphene sheets. The authors compared effects of both types of defects on material properties and concluded that vacancy defects cause more reduction the material properties as the Stone-Wales defects.

In the paper [4], the buckling characteristic of the defect-free graphene sheet while considering the influences of chirality, boundary conditions and geometrical parameters were explored. The buckling analysis of a defective annular graphene sheet was investigated by Fadaee [5]. The graphene sheet was modelled as a shell and the defect was modelled as an eccentric hole. It was observed that the eccentric defect has significant effect on the critical load.

In this study, the buckling analysis of graphene sheets is investigated. The graphene sheet is modelled using the finite element method. The interatomic interactions are modelled using beam elements and carbon atoms are modelled as nodes. On the graphene sheets are applied boundary conditions and the buckling analysis is performed. The analyses are performed for the sheets with and without defects. The critical buckling force is decreased for the increased number of defects in the structure.

\section{Modelling of graphene sheet}

The graphene sheets can be regarded in molecular mechanics as large molecules consisting of carbon atoms and the atomic nuclei as material points. The interatomic interactions between atoms are shown in Fig. 1.

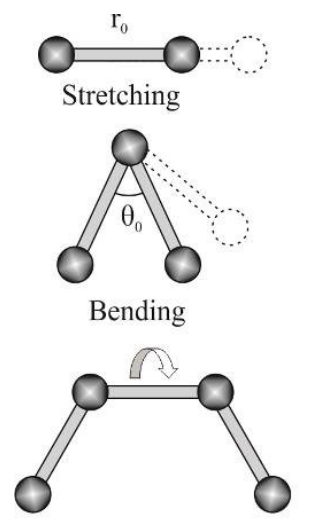

Dihedral angle torsion
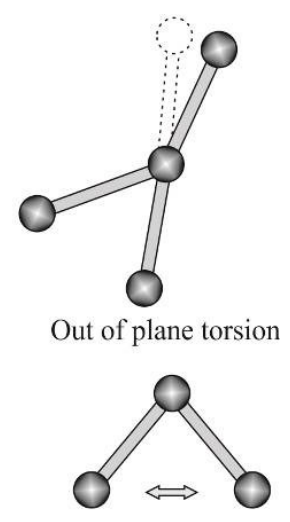

van der Waals

Fig. 1. Interatomic interactions in molecular mechanics

These interatomic interactions between carbon atoms can be expressed by relation

$$
U_{\text {total }}=1 \quad U_{r}+1 \quad U_{\mathrm{q}}+1 \quad U_{\mathrm{f}}+1 \quad U_{\mathrm{w}}+1 \quad U_{v d w},
$$

where $U_{r}, U_{\mathrm{q}}, U_{\mathrm{f}}, U_{\mathrm{w}}, U_{v d w}$ are a bond stretching, a bond angle bending, a dihedral angle torsion, an improper (out of plane) torsion and a non-bonded van der Waals interaction, respectively. These interatomic interactions can be modelled and represented in structural mechanics using beam elements. The material properties of the beam elements are computed in terms of the covalent bond stiffness to simulate the interatomic interactions. The properties are obtained from the comparison of the potential energies of the molecular and structural mechanics. Li and Chou [6] defined relations between structural and molecular parameters as 


$$
k_{r}=\frac{E A}{L}, k_{\mathrm{q}}=\frac{E I}{L}, k_{\mathrm{t}}=\frac{G J}{L},
$$

where $L$ is the beam length, $E$ is the Young's modulus, $G$ is the shear modulus, $I$ is the moment of inertia, $J$ is the polar moment of inertia, respectively. The constants $k_{r}, k_{\mathrm{q}}, k_{\mathrm{t}}$ are force constants (stiffness) of bond stretching, bond bending, and torsional resistance, respectively. If the interatomic interactions will be modelled by cylindrical beam elements (Fig. 2), we can obtain the input data for the finite element model. The carbon atoms will be modelled as the nodes of the beam elements. The all data are collected and shown in Table $1[6,7]$.

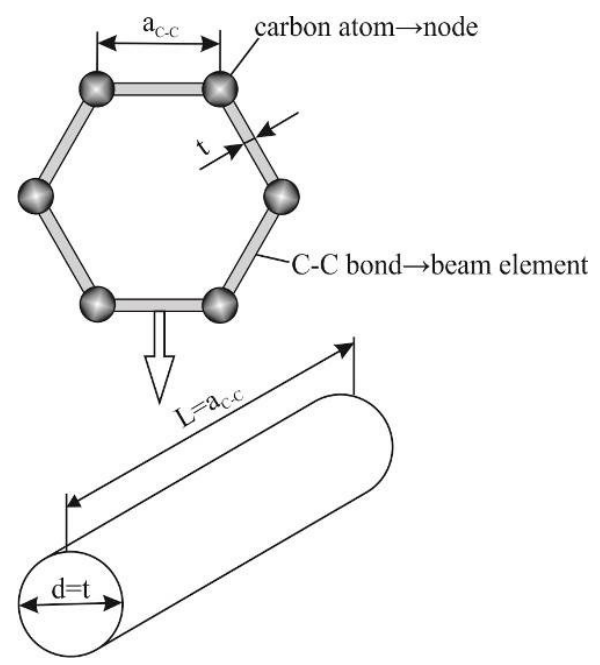

Fig. 2. Hexagon of modelled graphene sheet

Table 1. Basic and input parameters for graphene sheets

\begin{tabular}{|c|c|c|}
\hline Parameter & Symbol & Value \\
\hline Force constant & $k_{r}$ & $6.52 \times 10^{-7} \mathrm{Nnm}^{-1}$ \\
\hline Force constant & $k_{\theta}$ & $8.76 \times 10^{-10} \mathrm{Nnmrad}^{-2}$ \\
\hline Force constant & $k_{\tau}$ & $2.78 \times 10^{-10} \mathrm{Nnmrad}^{-2}$ \\
\hline Beam/C-C bond length & $\mathrm{L}=\mathrm{a}_{\mathrm{C}-\mathrm{C}}$ & $0.1421 \mathrm{~nm}$ \\
\hline Diameter & $\mathrm{d}=\mathrm{t}$ & $0.1466 \mathrm{~nm}$ \\
\hline Young's modulus & $\mathrm{E}$ & $5.488 \mathrm{TPa}$ \\
\hline Shear modulus & $\mathrm{G}$ & $0.871 \mathrm{TPa}$ \\
\hline
\end{tabular}

\section{Buckling of graphene sheet}

The buckling of graphene sheets is investigated in this chapter. At the first, the graphene sheets without defect are modelled and the buckling analysis is performed. The buckling analysis is performed for the two types of sheets with different types of internal structure (armchair and zigzag types) and lengths (from $2.5 \mathrm{~nm}$ to $50 \mathrm{~nm}$ ). The structure and dimensions of modelled graphene sheets are shown in Table 2. The chirality of the graphene sheet defines the size and types of edges of the graphene sheets. 
Table 2. Structure and dimensions of modelled graphene sheets

\begin{tabular}{|c|c|c|}
\hline Type of edge & Chirality & Width (nm) \\
\hline \multirow{3}{*}{ Armchair } & $(6,6)$ & 2.5578 \\
\cline { 2 - 3 } & $(8,8)$ & 3.2683 \\
\cline { 2 - 3 } & $(10,10)$ & 4.1209 \\
\hline \multirow{3}{*}{ Zigzag } & $(10,0)$ & 2.4612 \\
\cline { 2 - 3 } & $(13,0)$ & 3.1996 \\
\cline { 2 - 3 } & $(17,0)$ & 4.1841 \\
\hline
\end{tabular}

For these analyses two different boundary conditions are applied, the first type is represented as sheet fixed on one end and the second type is represented as sheet simply supported on two ends. In semi-logarithmic graph in Fig.4 are shown critical buckling forces for all modelled graphene sheets with the cantilever boundary conditions. The critical buckling forces for all modelled graphene sheet with the simply supported boundary conditions are shown in semi-logarithmic graph in Fig. 5.

From the figures is obvious that graphene sheets of armchair structure have a little bit higher buckling forces than the other structures.

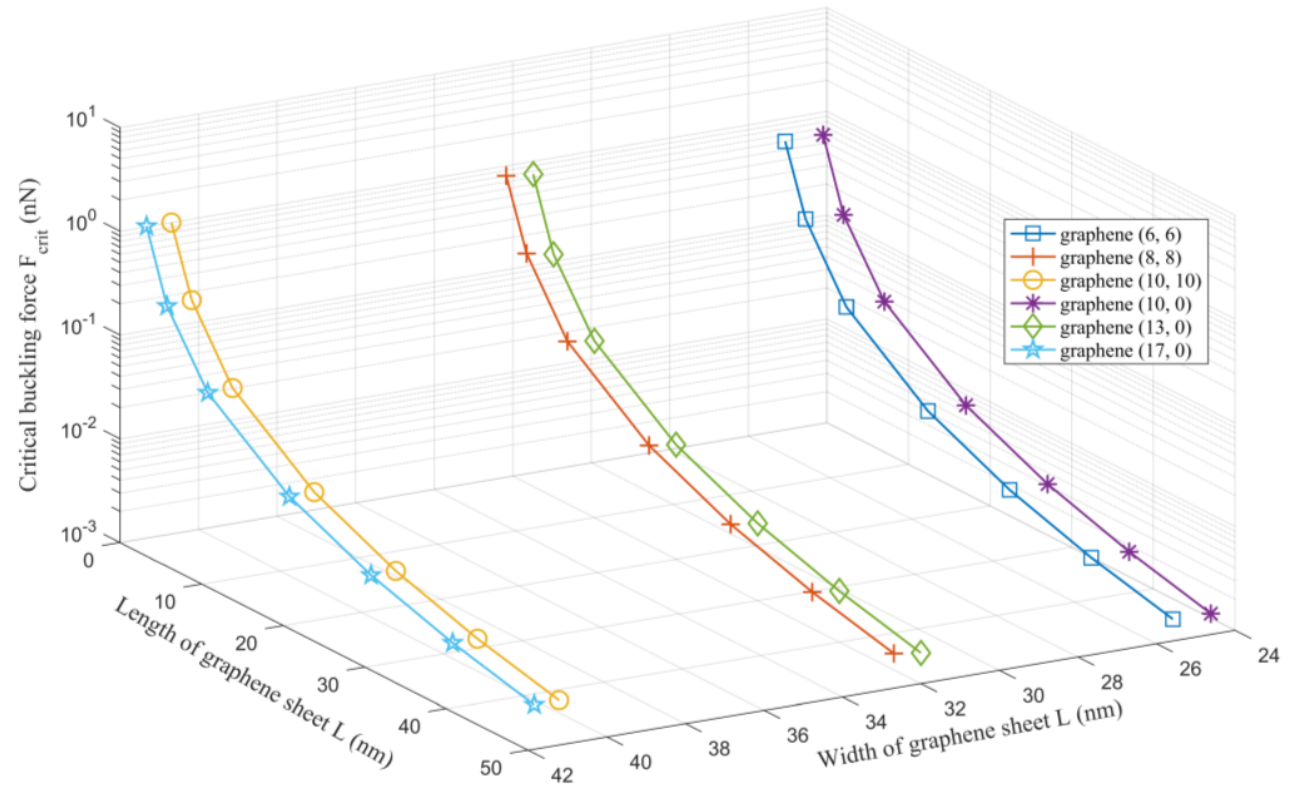

Fig. 4. Critical buckling force of graphene sheets (cantilever sheets) 


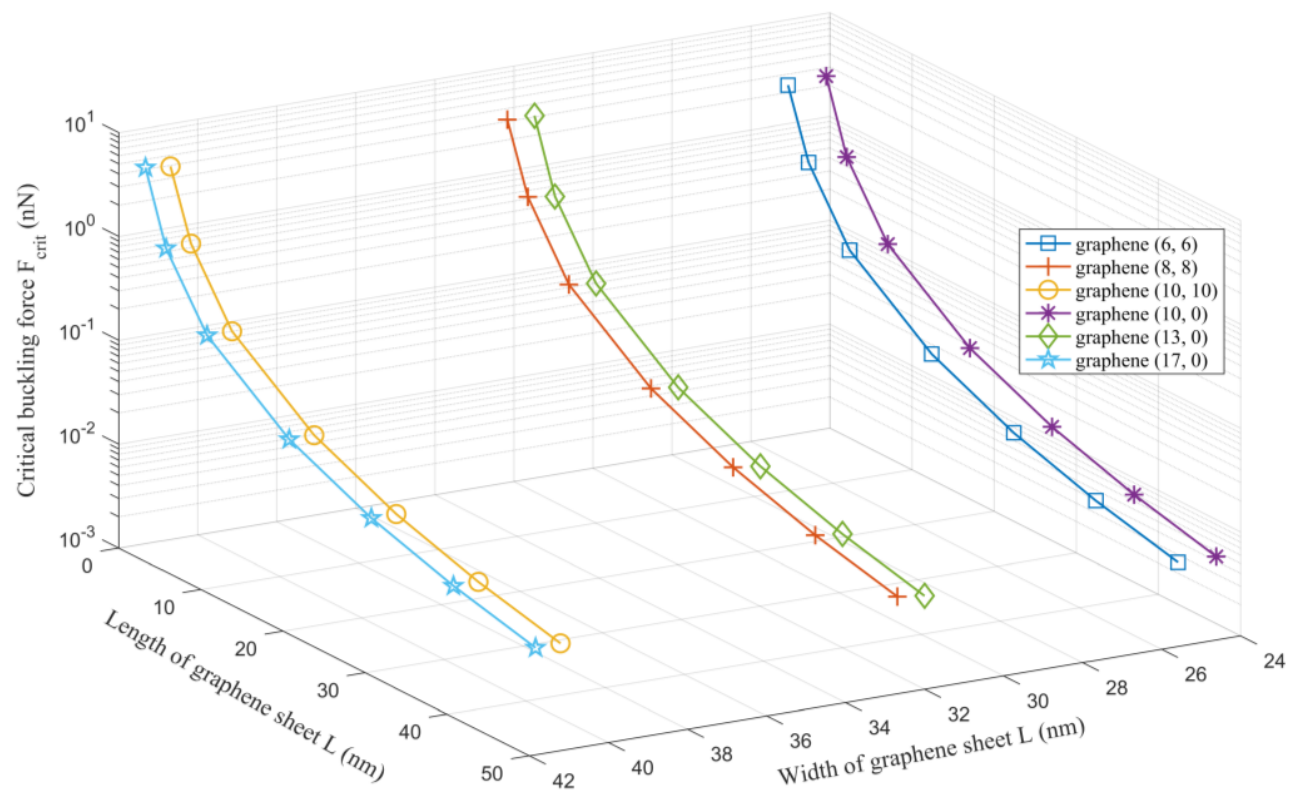

Fig. 5. Critical buckling force of graphene sheets (simply supported sheets)

The defected graphene sheets are modelled by removing random carbon atoms and beam elements connected to these atoms (nodes). From the sheet are removed 1\%, 3\% and $5 \%$ of carbon atoms from the total amount of atoms of ideal structure (Fig. 6). The effect of amount of defects on the critical buckling force is investigated. Dependencies of structural defects on buckling forces for different boundary conditions are given in Tables 3 (cantilever sheet) and 4 (simply supported sheet).

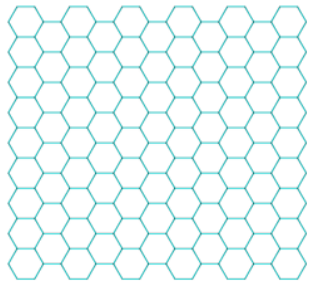

a)

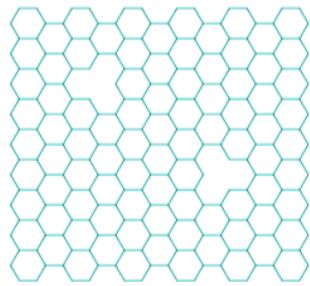

b)

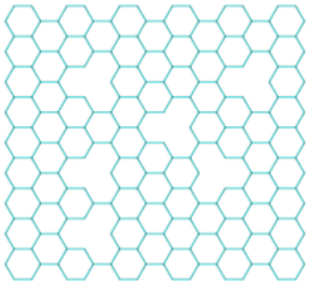

c)

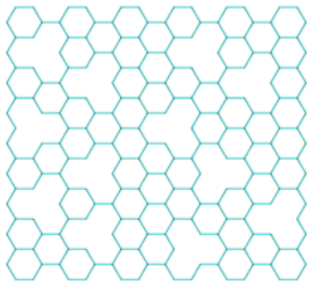

d)

Fig. 6. Modelled graphene sheets with a) $0 \%$, b) $1 \%$, c) $3 \%$ and d) $5 \%$ of defects

Table 3. Influence of structural defects on buckling force (cantilever sheet)

\begin{tabular}{|c|c|c|c|c|c|}
\hline \multicolumn{3}{|c|}{ Graphene sheet } & \multicolumn{3}{c|}{ Decrease of $\mathbf{F}_{\text {crit }}(\%)$} \\
\hline Type of edge & Chirality & Width (nm) & Defect 1\% & Defect 3\% & Defect 5\% \\
\hline \multirow{3}{*}{ Armchair } & $(6,6)$ & 2.5578 & 3.9011 & 11.0704 & 18.6456 \\
\cline { 2 - 6 } & $(8,8)$ & 3.2683 & 3.8570 & 10.8901 & 18.0937 \\
\cline { 2 - 6 } & $(10,10)$ & 4.1209 & 4.1043 & 11.3089 & 18.4064 \\
\hline \multirow{3}{*}{ Zigzag } & $(10,0)$ & 2.4612 & 4.2806 & 11.6256 & 18.7916 \\
\cline { 2 - 6 } & $(13,0)$ & 3.1996 & 4.0093 & 11.2019 & 18.6756 \\
\cline { 2 - 6 } & $(17,0)$ & 4.1841 & 3.9077 & 11.2548 & 18.3841 \\
\hline
\end{tabular}


Table 4. Influence of structural defects on buckling force (simply supported sheet)

\begin{tabular}{|c|c|c|c|c|c|}
\hline \multicolumn{3}{|c|}{ Graphene sheet } & \multicolumn{3}{c|}{ Decrease of $\mathbf{F}_{\text {crit }}(\%)$} \\
\hline Type of edge & Chirality & Width (nm) & Defect 1\% & Defect 3\% & Defect 5\% \\
\hline \multirow{3}{*}{ Armchair } & $(6,6)$ & 2.5578 & 4.1168 & 11.5566 & 18.8878 \\
\cline { 2 - 6 } & $(8,8)$ & 3.2683 & 4.0328 & 11.3523 & 18.2771 \\
\cline { 2 - 6 } & $(10,10)$ & 4.1209 & 3.9452 & 11.7698 & 18.9616 \\
\hline \multirow{3}{*}{ Zigzag } & $(10,0)$ & 2.4612 & 4.6785 & 11.7845 & 19.7319 \\
\cline { 2 - 6 } & $(13,0)$ & 3.1996 & 3.9811 & 11.6983 & 19.0965 \\
\cline { 2 - 6 } & $(17,0)$ & 4.1841 & 4.0355 & 11.7920 & 19.4753 \\
\hline
\end{tabular}

\section{Conclusion}

The buckling analysis of graphene sheets without and with defects was described in this paper. The graphene sheets with different types of chirality, sizes and boundary conditions were modelled by the finite element method. The interatomic interactions were modelled by beam elements and carbon atoms were modelled as joints of these beams. The input properties for the finite element model were obtained from connection between the molecular and structural mechanics. The computations were accomplished for the different percentage of atom vacancies $(0 \%, 1 \%, 3 \%, 5 \%)$ and the results represents an obvious fact that the critical buckling force decreases for increased percentage of vacancies in the structure. For the given percentage of errors, we have got buckling forces by 4, 11, 19 percent, respectively, smaller than those for ideal structures. The results were represented in form graphs and tables. The critical buckling force of the graphene sheets with armchair structure is a little bit higher than the buckling forces of other ones.

This research was supported by a grant from the Slovak Grant Agency VEGA No. 1/0731/16 Development of Modern Numerical and Experimental Methods of Mechanical System Analysis.

\section{References}

1. A. Sakhaee-Pour, Elastic properties of single-layered graphene sheet. Solid State Commun. 149, 1-2 (2009)

2. S.K. Georgantzinos, G.I. Giannopoulos, N.K. Anifantis, Numerical investigation of elastic mechanical properties of graphene structures. Mater. \& Design 31, 10 (2010)

3. R. Ansari, S. Ajori, B. Motevalli, Mechanical properties of defective single-layered graphene sheets via molecular dynamics simulation. Superlattices Microstruct. 51, 2 (2012)

4. A. Sakhaee-Pour, Elastic buckling of single-layered graphene sheet. Comp. Mater. Sci. 45, 2 (2009)

5. M. Fadaee, Buckling analysis of a defective annular graphene sheet in elastic medium. Appl. Math. Model. 40, 3 (2016)

6. Ch. Li, T. Chou, A structural mechanics approach for the analysis of carbon nanotubes. Int. J. Solid. Struct. 40, (2003)

7. J. Bocko, P. Lengvarský, Buckling of single-walled carbon nanotubes with and without defects. J. Mech. Sci. Technol. 31, 4 (2017) 